Journal of Universal Mathematics

VOL.4 No.1 PP.25-33 (2021)

ISSN-2618-5660

DOI: $10.33773 /$ jum. 876269

\title{
A STUDY ON GCR-LIGHTLIKE SUBMANIFOLDS OF SEMI-RIEMANNIAN PRODUCT MANIFOLDS
}

\author{
NERGIZ (ÖNEN) POYRAZ
}

\begin{abstract}
We study GCR-lightlike submanifolds of semi-Riemannian product manifolds. We give some equivalent conditions for the integrability of various distributions of GCR-lightlike submanifolds of semi-Riemannian product manifolds and investigate the geometry of leaves of distributions.
\end{abstract}

\section{INTRODUCTION}

The theory of lightlike submanifolds is an important research topic in differential geometry due to its application in mathematical physics, especially in the general relativity. Since the normal vector bundle intersects with the tangent bundle, contrary to classical theory of submanifolds, the theory of lightlike (degenerate) submanifolds becomes more interesting and remarkably different from the theory of non-degenerate submanifolds. The geometry of lightlike submanifolds was initiated by Kupeli [16]. After it was developed by Duggal and Bejancu [3] and Duggal and Sahin [7]. Many authors studied the lightlike submanifolds in various manifolds, for example [2], [11], [18] and [20].

Duggal and Bejancu introduced CR-lightlike submanifolds of indefinite Kaehler manifolds [3]. Similar to CR-lightlike submanifolds, semi-invariant lightlike submanifolds of semi-Riemannian product manifolds were introduced by Atçeken and Kılıç in [1]. Since CR-lightlike submanifolds exclude the complex and totally real submanifolds, therefore, in [4], Duggal and Şahin introduced screen CR-lightlike submanifolds, which contains complex and screen real subcases. The SCR-lightlike submanifolds, analogously, Screen semi-invariant lightlike submanifolds, of semiRiemannian product manifolds were introduced by Khursheed, Thakur and Advin [10] and Kılıç, Şahin and Keleş [12], respectively. But there is no inclusion relation between CR and SCR submanifolds, so Duggal and Şahin introduced a new class called GCR-lightlike submanifolds of indefinite Kaehler manifolds which is an umbrella for all these types of submanifolds [5]. Kumar, Kumar, Nagaich studied GCR-lightlike submanifolds of a semi-Riemannian product manifold [15]. These

Date: February, 2021.

2000 Mathematics Subject Classification. 53C15, 53C40, 53C50.

Key words and phrases. Semi-Riemannian product manifolds, Lightlike submanifolds, GCRlightlike submanifolds. 
types of submanifolds have been studied in various manifolds by many authors $[6,8,9,13,14,17]$.

In this paper, we study GCR-lightlike submanifolds of semi-Riemannian product manifolds. We give some equivalent conditions for the integrability of various distributions of GCR-lightlike submanifolds of semi-Riemannian product manifolds and investigate the geometry of leaves of distributions.

\section{Preliminaries}

Let $(\tilde{M}, \tilde{g})$ be a real $(m+n)$-dimensional semi-Riemannian manifold of constant index $q$, such that $m, n \geq 1,1 \leq q \leq m+n-1$ and $(M, g)$ be an $m$-dimensional submanifold of $\tilde{M}$, where $g$ is the induced metric of $\tilde{g}$ on $M$. If $\tilde{g}$ is degenerate on the tangent bundle $T M$ of $M$ then $M$ is called a lightlike submanifold of $\tilde{M}$. For a degenerate metric $g$ on $M$

$$
T M^{\perp}=\cup\left\{u \in T_{x} \tilde{M}: \tilde{g}(u, v)=0, \forall v \in T_{x} M, x \in M\right\}
$$

is a degenerate $n$-dimensional subspace of $T_{x} \tilde{M}$. Thus, both $T_{x} M$ and $T_{x} M^{\perp}$ are degenerate orthogonal subspaces but no longer complementary. In this case, there exists a subspace $\operatorname{Rad}\left(T_{x} M\right)=T_{x} M \cap T_{x} M^{\perp}$ which is known as radical (null) space. If the mapping $\operatorname{Rad}(T M): x \in M \longrightarrow \operatorname{Rad}\left(T_{x} M\right)$, defines a smooth distribution, called radical distribution on $M$ of rank $r>0$ then the submanifold $M$ of $\tilde{M}$ is called an $r$-lightlike submanifold.

Let $S(T M)$ be a screen distribution which is a semi-Riemannian complementary distribution of $\operatorname{Rad}(T M)$ in $T M$. This means that

$$
T M=S(T M) \perp \operatorname{Rad}(T M)
$$

and $S\left(T M^{\perp}\right)$ is a complementary vector subbundle to $\operatorname{Rad}(T M)$ in $T M^{\perp}$. Let $\operatorname{tr}(T M)$ and $\operatorname{lt}(T M)$ be complementary (but not orthogonal) vector bundles to $T M$ in $T \tilde{M}_{\left.\right|_{M}}$ and $\operatorname{Rad}(T M)$ in $S\left(T M^{\perp}\right)^{\perp}$, respectively. Then, we have

$$
\operatorname{tr}(T M)=\operatorname{ltr}(T M) \perp S\left(T M^{\perp}\right),
$$

$$
\left.T \tilde{M}\right|_{M}=T M \oplus \operatorname{tr}(T M)=\{\operatorname{Rad}(T M) \oplus \operatorname{ltr}(T M)\} \perp S(T M) \perp S\left(T M^{\perp}\right) .
$$

Theorem 2.1. [3] Let $\left(M, g, S(T M), S\left(T M^{\perp}\right)\right)$ be an $r$-lightlike submanifold of a semi-Riemannian manifold $(\tilde{M}, \tilde{g})$. Suppose $U$ is a coordinate neighbourhood of $M$ and $\left\{\xi_{i}\right\}, i \in\{1, . ., r\}$ is a basis of $\Gamma\left(\operatorname{Rad}(T M)_{\left.\right|_{U}}\right)$. Then, there exist a complementary vector subbundle ltr $(T M)$ of $\operatorname{Rad}(T M)$ in $\left.S\left(T M^{\perp}\right)\right|_{\left.\right|_{U}} ^{\perp}$ and a basis $\left\{N_{i}\right\}, i \in\{1, . ., r\}$ of $\Gamma\left(\operatorname{ltr}(T M)_{\left.\right|_{U}}\right)$ such that

$$
\tilde{g}\left(N_{i}, \xi_{j}\right)=\delta_{i j}, \quad \tilde{g}\left(N_{i}, N_{j}\right)=0
$$

for any $i, j \in\{1, . ., r\}$.

We say that a submanifold $\left(M, g, S(T M), S\left(T M^{\perp}\right)\right)$ of $\tilde{M}$ is

Case 1: $r$-lightlike if $r<\min \{m, n\}$,

Case 2: Coisotropic if $r=n<m ; S\left(T M^{\perp}\right)=\{0\}$,

Case 3: Isotropic if $r=m<n ; S(T M)=\{0\}$,

Case 4: Totally lightlike if $r=m=n ; S(T M)=\{0\}=S\left(T M^{\perp}\right)$. 
Let $\tilde{\nabla}$ be the Levi-Civita connection on $\tilde{M}$. Then, using (2.4), the Gauss and Weingarten formulas are given by

$$
\begin{aligned}
\tilde{\nabla}_{X} Y & =\nabla_{X} Y+h(X, Y), \\
\tilde{\nabla}_{X} U & =-A_{U} X+\nabla_{X}^{t} U,
\end{aligned}
$$

for any $X, Y \in \Gamma(T M)$ and $U \in \Gamma(\operatorname{tr}(T M))$, where $\left\{\nabla_{X} Y, A_{U} X\right\}$ and $\left\{h(X, Y), \nabla_{X}^{t} U\right\}$ belong to $\Gamma(T M)$ and $\Gamma(\operatorname{tr}(T M))$, respectively. $\nabla$ and $\nabla^{t}$ are linear connections on $M$ and on the vector bundle $\operatorname{tr}(T M)$, respectively. According to (2.3), considering the projection morphisms $L$ and $S$ of $\operatorname{tr}(T M)$ on $\operatorname{ltr}(T M)$ and $S\left(T M^{\perp}\right)$, respectively, (2.6) and (2.7) become

$$
\begin{aligned}
\tilde{\nabla}_{X} Y & =\nabla_{X} Y+h^{l}(X, Y)+h^{s}(X, Y), \\
\tilde{\nabla}_{X} N & =-A_{N} X+\nabla_{X}^{l} N+D^{s}(X, N), \\
\tilde{\nabla}_{X} W & =-A_{W} X+\nabla_{X}^{s} W+D^{l}(X, W),
\end{aligned}
$$

for any $X, Y \in \Gamma(T M), N \in \Gamma(l \operatorname{tr}(T M))$ and $W \in \Gamma\left(S\left(T M^{\perp}\right)\right)$, where $h^{l}(X, Y)=$ $L h(X, Y), h^{s}(X, Y)=S h(X, Y), \nabla_{X} Y, A_{N} X, A_{W} X \in \Gamma(T M), \nabla_{X}^{l} N, D^{l}(X, W) \in$ $\Gamma(l \operatorname{tr}(T M))$ and $\nabla_{X}^{s} W, D^{s}(X, N) \in \Gamma\left(S\left(T M^{\perp}\right)\right)$. Then, by using (2.8)-(2.10) and taking into account that $\tilde{\nabla}$ is a metric connection we obtain

$$
\begin{aligned}
\tilde{g}\left(h^{s}(X, Y), W\right)+\tilde{g}\left(Y, D^{l}(X, W)\right) & =g\left(A_{W} X, Y\right), \\
\tilde{g}\left(D^{s}(X, N), W\right) & =\tilde{g}\left(A_{W} X, N\right) .
\end{aligned}
$$

Let $Q$ be a projection of $T M$ on $S(T M)$. Then, using (2.2) we can write

$$
\begin{aligned}
\nabla_{X} Q Y & =\nabla_{X}^{*} Q Y+h^{*}(X, Q Y), \\
\nabla_{X} \xi & =-A_{\xi}^{*} X+\nabla_{X}^{* t} \xi,
\end{aligned}
$$

for any $X, Y \in \Gamma(T M)$ and $\xi \in \Gamma(\operatorname{Rad}(T M))$, where $\left\{\nabla_{X}^{*} Q Y, A_{\xi}^{*} X\right\}$ and $\left\{h^{*}(X, Q Y), \nabla_{X}^{* t} \xi\right\}$ belong to $\Gamma(S(T M))$ and $\Gamma(\operatorname{Rad}(T M))$, respectively.

Using the equations given above, we derive

$$
\begin{aligned}
\tilde{g}\left(h^{l}(X, Q Y), \xi\right) & =g\left(A_{\xi}^{*} X, Q Y\right), \\
\tilde{g}\left(h^{*}(X, Q Y), N\right) & =g\left(A_{N} X, Q Y\right), \\
\tilde{g}\left(h^{l}(X, \xi), \xi\right) & =0, \quad A_{\xi}^{*} \xi=0 .
\end{aligned}
$$

Generally, the induced connection $\nabla$ on $M$ is not metric connection. Since $\tilde{\nabla}$ is a metric connection, from (2.8), we obtain

$$
\left(\nabla_{X} g\right)(Y, Z)=\tilde{g}\left(h^{l}(X, Y), Z\right)+\tilde{g}\left(h^{l}(X, Z), Y\right) .
$$

But, $\nabla^{*}$ is a metric connection on $S(T M)$.

\section{Semi-Riemannian PRoduct Manifolds}

Let $\left(M_{1}, g_{1}\right)$ and $\left(M_{2}, g_{2}\right)$ be two $\left(m_{1}+1\right)$ and $\left(m_{2}+1\right)$ dimensional Lorentzian manifolds with constant indexes $q_{1}>0, q_{2}>0$, respectively. Let $\pi: M_{1} \times M_{2} \rightarrow$ $M_{1}$ and $\sigma: M_{1} \times M_{2} \rightarrow M_{2}$ be the projections which are given by $\pi(x, y)=x$ and $\sigma(x, y)=y$ for any $(x, y) \in M_{1} \times M_{2}$. We denote the product manifold by $\tilde{M}=M_{1} \times M_{2}$, where

$$
\tilde{g}(X, Y)=g_{1}\left(\pi_{*} X, \pi_{*} Y\right)+g_{2}\left(\sigma_{*} X, \sigma_{*} Y\right),
$$

for any $X, Y \in \Gamma(T \tilde{M})$ and $*$ means tangent mapping. Then we have $\pi_{*}^{2}=\pi$, $\sigma_{*}^{2}=\sigma, \pi_{*} \sigma_{*}=\sigma_{*} \pi_{*}=0$ and $\pi_{*}+\sigma_{*}=I$, where $I$ is identity transformation. Thus 
$(\tilde{M}, \tilde{g})$ is a $\left(m_{1}+m_{2}\right)$-dimensional semi-Riemannian manifold with constant index $\left(q_{1}+q_{2}\right)$. The semi-Riemannian product manifold $\tilde{M}=M_{1} \times M_{2}$ is characterized by $M_{1}$ and $M_{2}$ which are totally geodesic submanifolds of $\tilde{M}$.

Now, if we put $F=\pi_{*}-\sigma_{*}$, then we can easily see that

$$
F^{2}=I
$$

and

$$
\tilde{g}(F X, Y)=\tilde{g}(X, F Y)
$$

for any $X, Y \in \Gamma(T \tilde{M})$. Then it can be seen that

$$
\left(\tilde{\nabla}_{X} F\right) Y=0
$$

for any $X, Y \in \Gamma(T \tilde{M})$, that is, $F$ is parallel with respect to $\tilde{\nabla}$ [19].

\section{Generalized Cauchy-Riemann (GCR)-Lightlike Submanifolds}

Definition 4.1. Let $(M, g, S(T M))$ be a real lightlike submanifold of a semiRiemannian product manifold $(\tilde{M}, \tilde{g}, F)$. Then we say that $M$ is a generalized Cauchy-Riemann (GCR)-lightlike submanifold if the following conditions are satisfied:

(A) There exist two subbundles $D_{1}$ and $D_{2}$ of $\operatorname{Rad}(T M)$ such that

$$
\operatorname{Rad}(T M)=D_{1} \oplus D_{2}, F\left(D_{1}\right)=D_{1}, F\left(D_{2}\right) \subset S(T M) .
$$

(B) There exist two subbundles $D_{0}$ and $D^{\prime}$ of $S(T M)$ such that

$$
S(T M)=\left\{F\left(D_{2}\right) \oplus D^{\prime}\right\} \perp D_{0}, F\left(D_{0}\right)=D_{0}, F\left(D^{\prime}\right)=L_{1} \perp L_{2},
$$

where $D_{0}$ is a non-degenerate distribution on $M, L_{1}$ and $L_{2}$ are vector subbundles of $l \operatorname{tr}(T M)$ and $S\left(T M^{\perp}\right)$, respectively.

Thus we have the following decomposition:

$$
T M=D \oplus D^{\prime}, D=\operatorname{Rad}(T M) \perp D_{0} \perp F\left(D_{2}\right) .
$$

We say that $M$ is a proper GCR-lightlike submanifold of a semi-Riemannian product manifold if $D_{0} \neq\{0\}, D_{1} \neq\{0\}, D_{2} \neq\{0\}$ and $L_{2} \neq\{0\}$.

Let $M$ be a GCR-lightlike submanifold of a semi-Riemannian product manifold $(\tilde{M}, \tilde{g}, F)$. Thus, for any $X \in \Gamma(T M)$ we derive

$$
F X=f X+w X,
$$

where $f X$ and $w X$ are tangential and transversal parts of $F X$.

For $V \in \Gamma(\operatorname{tr}(T M))$ we write

$$
F V=B V+C V,
$$

where $B V$ and $C V$ are tangential and transversal parts of $F V$.

Theorem 4.2. Let $M$ be a GCR-lightlike submanifold of a semi-Riemannian product manifold $(\tilde{M}, \tilde{g}, F)$. Then the distribution $D_{0}$ is integrable iff

(i) $\tilde{g}\left(h^{*}(X, Y), N\right)=\tilde{g}\left(h^{*}(Y, X), N\right)$,

(ii) $\tilde{g}\left(h^{*}(X, F Y), N^{\prime}\right)=\tilde{g}\left(h^{*}(Y, F X), N^{\prime}\right)$,

(iii) $\tilde{g}\left(h^{s}(X, F Y), W\right)=\tilde{g}\left(h^{s}(Y, F X), W\right)$,

(iv) $g\left(\nabla_{X}^{*} Y, F \xi\right)=g\left(\nabla_{Y}^{*} X, F \xi\right)$,

for any $X, Y \in \Gamma\left(D_{0}\right), \xi \in \Gamma\left(D_{2}\right), N \in \Gamma(\operatorname{ltr}(T M)), N^{\prime} \in \Gamma\left(L_{1}\right)$ and $W \in$ $\Gamma\left(L_{2}\right)$. 
Proof. Using the definition of GCR-lightlike submanifold, the distribution $D_{0}$ is integrable iff

$$
\tilde{g}([X, Y], N)=\tilde{g}\left([X, Y], F N^{\prime}\right)=\tilde{g}([X, Y], F W)=\tilde{g}([X, Y], F \xi)=0 .
$$

for any $X, Y \in \Gamma\left(D_{0}\right), \xi \in \Gamma\left(D_{2}\right), N \in \Gamma(l \operatorname{tr}(T M)), N^{\prime} \in \Gamma\left(L_{1}\right)$ and $W \in \Gamma\left(L_{2}\right)$. Then using (2.8) and (2.13) we obtain

$$
\begin{aligned}
\tilde{g}([X, Y], N) & =\tilde{g}\left(\tilde{\nabla}_{X} Y-\tilde{\nabla}_{Y} X, N\right)=\tilde{g}\left(h^{*}(X, Y)-h^{*}(Y, X), N\right), \\
g\left([X, Y], F N^{\prime}\right) & =g\left(\tilde{\nabla}_{X} Y-\tilde{\nabla}_{Y} X, F N^{\prime}\right)=\tilde{g}\left(\tilde{\nabla}_{X} F Y-\tilde{\nabla}_{Y} F X, N^{\prime}\right) \\
& =\tilde{g}\left(h^{*}(X, F Y)-h^{*}(Y, F X), N^{\prime}\right), \\
g([X, Y], F W) & =g\left(\tilde{\nabla}_{X} Y-\tilde{\nabla}_{Y} X, F W\right)=\tilde{g}\left(\tilde{\nabla}_{X} F Y-\tilde{\nabla}_{Y} F X, W\right) \\
& =\tilde{g}\left(h^{s}(X, F Y)-h^{s}(Y, F X), W\right), \\
g([X, Y], F \xi) & =g\left(\tilde{\nabla}_{X} Y-\tilde{\nabla}_{Y} X, F \xi\right)=g\left(\nabla_{X}^{*} Y, F \xi\right)-g\left(\nabla_{Y}^{*} X, F \xi\right) .
\end{aligned}
$$

Thus from (4.6)-(4.9) the result follows.

From Theorem 4.2 we obtain the following corollary.

Corollary 4.3. Let $M$ be a GCR-lightlike submanifold of a semi-Riemannian product manifold $(\tilde{M}, \tilde{g}, F)$. Then the distribution $D_{0}$ is integrable iff

(i) $g\left(A_{N} X, Y\right)=g\left(A_{N} Y, X\right)$

(ii) $g\left(A_{N^{\prime}} X, F Y\right)=g\left(A_{N^{\prime}} Y, F X\right)$,

(iii) $g\left(A_{W} X, F Y\right)=g\left(A_{W} Y, F X\right)$,

(iv) $\tilde{g}\left(h^{l}(X, F Y), \xi\right)=\tilde{g}\left(h^{l}(Y, F X), \xi\right)$,

for any $X, Y \in \Gamma\left(D_{0}\right), \xi \in \Gamma\left(D_{2}\right), N \in \Gamma(\operatorname{ltr}(T M)), N^{\prime} \in \Gamma\left(L_{1}\right)$ and $W \in \Gamma\left(L_{2}\right)$.

Theorem 4.4. Let $M$ be a GCR-lightlike submanifold of a semi-Riemannian product manifold $(\tilde{M}, \tilde{g}, F)$. Then $\operatorname{Rad}(T M)$ is integrable iff

(i) $\tilde{g}\left(h^{l}(\xi, Z), \xi^{\prime}\right)=\tilde{g}\left(h^{l}\left(\xi^{\prime}, Z\right), \xi\right)$,

(ii) $\tilde{g}\left(h^{l}\left(\xi, F \xi^{\prime \prime}\right), \xi^{\prime}\right)=\tilde{g}\left(h^{l}\left(\xi^{\prime}, F \xi^{\prime \prime}\right), \xi\right)$,

(iii) $\tilde{g}\left(h^{*}\left(\xi, F \xi^{\prime}\right), N\right)=\tilde{g}\left(h^{*}\left(\xi^{\prime}, F \xi\right), N\right)$,

(iv) $\tilde{g}\left(h^{s}\left(\xi, F \xi^{\prime}\right), W\right)=\tilde{g}\left(h^{s}\left(\xi^{\prime}, F \xi\right), W\right)$,

for any $Z \in \Gamma\left(D_{0}\right), \xi^{\prime \prime} \in \Gamma\left(D_{2}\right), \xi, \xi^{\prime} \in \Gamma(\operatorname{Rad}(T M)), N \in \Gamma\left(L_{1}\right), W \in \Gamma\left(L_{2}\right)$.

Proof. Using the definition of GCR-lightlike submanifold, $\operatorname{Rad}(T M)$ is integrable iff

$$
\tilde{g}\left(\left[\xi, \xi^{\prime}\right], Z\right)=\tilde{g}\left(\left[\xi, \xi^{\prime}\right], F \xi^{\prime \prime}\right)=\tilde{g}\left(\left[\xi, \xi^{\prime}\right], F N\right)=\tilde{g}\left(\left[\xi, \xi^{\prime}\right], F W\right)=0,
$$

for any $Z \in \Gamma\left(D_{0}\right), \xi^{\prime \prime} \in \Gamma\left(D_{2}\right), \xi, \xi^{\prime} \in \Gamma(\operatorname{Rad}(T M)), N \in \Gamma\left(L_{1}\right), W \in \Gamma\left(L_{2}\right)$. Then taking into account that $\tilde{\nabla}$ is a metric connection and using (2.8) and (2.13) we get

$$
\begin{aligned}
g\left(\left[\xi, \xi^{\prime}\right], Z\right) & =g\left(\tilde{\nabla}_{\xi} \xi^{\prime}, Z\right)-g\left(\tilde{\nabla}_{\xi^{\prime}} \xi, Z\right)=-g\left(\xi^{\prime}, \tilde{\nabla}_{\xi} Z\right)+g\left(\xi, \tilde{\nabla}_{\xi^{\prime}} Z\right) \\
& =-\tilde{g}\left(h^{l}(\xi, Z), \xi^{\prime}\right)+\tilde{g}\left(h^{l}\left(\xi^{\prime}, Z\right), \xi\right),
\end{aligned}
$$

$$
g\left(\left[\xi, \xi^{\prime}\right], F \xi^{\prime \prime}\right)=g\left(\tilde{\nabla}_{\xi} \xi^{\prime}, F \xi^{\prime \prime}\right)-g\left(\tilde{\nabla}_{\xi^{\prime}} \xi, F \xi^{\prime \prime}\right)=-\tilde{g}\left(\xi^{\prime}, \tilde{\nabla}_{\xi} F \xi^{\prime \prime}\right)+\tilde{g}\left(\xi, \tilde{\nabla}_{\xi^{\prime}} F \xi^{\prime \prime}\right)
$$

$$
=-\tilde{g}\left(h^{l}\left(\xi, F \xi^{\prime \prime}\right), \xi^{\prime}\right)+\tilde{g}\left(h^{l}\left(\xi^{\prime}, F \xi^{\prime \prime}\right), \xi\right),
$$

$$
\tilde{g}\left(\left[\xi, \xi^{\prime}\right], F N\right)=g\left(\tilde{\nabla}_{\xi} \xi^{\prime}, F N\right)-g\left(\tilde{\nabla}_{\xi^{\prime}} \xi, F N\right)=g\left(\tilde{\nabla}_{\xi} F \xi^{\prime}, N\right)-g\left(\tilde{\nabla}_{\xi^{\prime}} F \xi, N\right)
$$

$$
=\tilde{g}\left(h^{*}\left(\xi, F \xi^{\prime}\right), N\right)-\tilde{g}\left(h^{*}\left(\xi^{\prime}, F \xi\right), N\right),
$$




$$
\begin{aligned}
g\left(\left[\xi, \xi^{\prime}\right], F W\right) & =g\left(\tilde{\nabla}_{\xi} \xi^{\prime}, F W\right)-g\left(\tilde{\nabla}_{\xi^{\prime}} \xi, F W\right)=\tilde{g}\left(\tilde{\nabla}_{\xi} F \xi^{\prime}, W\right)-\tilde{g}\left(\tilde{\nabla}_{\xi^{\prime}} F \xi, W\right) \\
(4.13) & =\tilde{g}\left(h^{s}\left(\xi, F \xi^{\prime}\right), W\right)-\tilde{g}\left(h^{s}\left(\xi^{\prime}, F \xi\right), W\right) .
\end{aligned}
$$

Hence proof is complete.

From Theorem 4.4 we get the following corollary.

Corollary 4.5. Let $M$ be a GCR-lightlike submanifold of a semi-Riemannian product manifold $(\tilde{M}, \tilde{g}, F)$. Then $\operatorname{Rad}(T M)$ is integrable iff

(i) $g\left(A_{\xi^{\prime}}^{*} \xi, Z\right)=g\left(A_{\xi}^{*} \xi^{\prime}, Z\right)$,

(ii) $g\left(A_{\xi^{\prime}}^{*} \xi, F \xi^{\prime \prime}\right)=g\left(A_{\xi}^{*} \xi^{\prime}, F \xi^{\prime \prime}\right)$,

(iii) $g\left(A_{N} \xi, F \xi^{\prime}\right)=g\left(A_{N} \xi^{\prime}, F \xi\right)$,

(iv) $g\left(A_{W} \xi, F \xi^{\prime}\right)=g\left(A_{W} \xi^{\prime}, F \xi\right)$,

for any $Z \in \Gamma\left(D_{0}\right), \xi^{\prime \prime} \in \Gamma\left(D_{2}\right), \xi, \xi^{\prime} \in \Gamma(\operatorname{Rad}(T M)), N \in \Gamma\left(L_{1}\right), W \in \Gamma\left(L_{2}\right)$.

Theorem 4.6. Let $M$ be a GCR-lightlike submanifold of a semi-Riemannian product manifold $(\tilde{M}, \tilde{g}, F)$. Then, each leaf of radical distribution is totally geodesic on $M$ iff

(i) $A_{\xi^{\prime}}^{*} \xi \in \Gamma\left(F\left(D_{2}\right) \perp F\left(L_{1}\right)\right)$,

(ii) $\tilde{g}\left(h^{*}(\xi, F N), \xi^{\prime}\right)=0$,

(iii) $\tilde{g}\left(h^{s}\left(\xi, F \xi^{\prime}\right), W\right)=0$,

for any $Z \in \Gamma\left(D_{0}\right), \xi, \xi^{\prime} \in \Gamma(\operatorname{Rad}(T M)), N \in \Gamma\left(L_{1}\right), W \in \Gamma\left(L_{2}\right)$.

Proof. Using the definition of GCR-lightlike submanifold, each leaf of radical distribution is totally geodesic iff

$$
g\left(\nabla_{\xi} \xi^{\prime}, Z\right)=g\left(\nabla_{\xi} \xi^{\prime}, F \xi^{\prime \prime}\right)=g\left(\nabla_{\xi} \xi^{\prime}, F N\right)=g\left(\nabla_{\xi} \xi^{\prime}, F W\right)=0,
$$

for any $Z \in \Gamma\left(D_{0}\right), \xi^{\prime \prime} \in \Gamma\left(D_{2}\right), \xi, \xi^{\prime} \in \Gamma(\operatorname{Rad}(T M)), N \in \Gamma\left(L_{1}\right), W \in \Gamma\left(L_{2}\right)$. Then taking into account that $\tilde{\nabla}$ is a metric connection and using (2.8) and (2.13), (2.14), we derive

$$
\begin{gathered}
g\left(\nabla_{\xi} \xi^{\prime}, Z\right)=g\left(\tilde{\nabla}_{\xi} \xi^{\prime}, Z\right)=-g\left(A_{\xi^{\prime}}^{*} \xi, Z\right), \\
g\left(\nabla_{\xi} \xi^{\prime}, F \xi^{\prime \prime}\right)=g\left(\tilde{\nabla}_{\xi} \xi^{\prime}, F \xi^{\prime \prime}\right)=-g\left(A_{\xi^{\prime}}^{*} \xi, F \xi^{\prime \prime}\right), \\
g\left(\nabla_{\xi} \xi^{\prime}, F N\right)=g\left(\tilde{\nabla}_{\xi} \xi^{\prime}, F N\right)=-\tilde{g}\left(\xi^{\prime}, \tilde{\nabla}_{\xi} F N\right)=-\tilde{g}\left(h^{*}(\xi, F N), \xi^{\prime}\right), \\
g\left(\nabla_{\xi} \xi^{\prime}, F W\right)=g\left(\tilde{\nabla}_{\xi} \xi^{\prime}, F W\right)=\tilde{g}\left(\tilde{\nabla}_{\xi} F \xi^{\prime}, W\right)=\tilde{g}\left(h^{s}\left(\xi, F \xi^{\prime}\right), W\right) .
\end{gathered}
$$

Hence from (4.14)-(4.17) the assertion follows.

Theorem 4.7. Let $M$ be a GCR-lightlike submanifold of a semi-Riemannian product manifold $(\tilde{M}, \tilde{g}, F)$. Then the distribution $D_{1}$ is integrable iff

(i) $\nabla_{X}^{* t} F Y-\nabla_{Y}^{* t} F X \in \Gamma\left(D_{1}\right)$,

(ii) $A_{F X}^{*} Y=A_{F Y}^{*} X$,

(iii) $B h(X, F Y)=B h(Y, F X)$,

for any $X, Y \in \Gamma\left(D_{1}\right)$.

Proof. For any $X, Y \in \Gamma(\operatorname{Rad}(T M))$ using (3.3), we have $\tilde{\nabla}_{X} F Y=F \tilde{\nabla}_{X} Y$, applying $F$ to both sides and then using (2.6), (2.14) and (3.1), we obtain

$$
\nabla_{X} Y+h(X, Y)=F\left(-A_{F Y}^{*} X+\nabla_{X}^{* t} F Y+h(X, F Y)\right),
$$


for any $X, Y \in \Gamma\left(D_{1}\right)$. Taking the tangential components of above equation both sides, we get

$$
\nabla_{X} Y=-f A_{F Y}^{*} X+f \nabla_{X}^{* t} F Y-B h(X, F Y)
$$

for any $X, Y \in \Gamma\left(D_{1}\right)$. Replacing $X$ by $Y$ and subtracting resulting equation from this equation, we derive

$$
\begin{aligned}
{[X, Y]=} & f\left(A_{F X}^{*} Y-A_{F Y}^{*} X\right)+f\left(\nabla_{X}^{* t} F Y-\nabla_{Y}^{* t} F X\right) \\
& -B h(X, F Y)+B h(Y, F X)
\end{aligned}
$$

thus $[X, Y] \in \Gamma\left(D_{1}\right)$ iff $\nabla_{X}^{* t} F Y-\nabla_{Y}^{* t} F X \in \Gamma\left(D_{1}\right), B h(X, F Y)=B h(Y, F X)$, $A_{F X}^{*} Y=A_{F Y}^{*} X$, this completes the proof.

From Theorem 4.7 we obtain the following corollary.

Corollary 4.8. Let $M$ be a GCR-lightlike submanifold of a semi-Riemannian product manifold $(\tilde{M}, \tilde{g}, F)$. Then $D_{1}$ defines a totally geodesic foliation in $M$ iff

(i) $\nabla_{X}^{* t} F Y \in \Gamma\left(D_{1}\right)$,

(ii) $A_{F X}^{*} Y=0$,

(iii) $B h(X, F Y)=0$,

for any $X, Y \in \Gamma\left(D_{1}\right)$.

Theorem 4.9. Let $M$ be a GCR-lightlike submanifold of a semi-Riemannian product manifold $(\tilde{M}, \tilde{g}, F)$. Then the distribution $D_{2}$ is integrable iff

(i) $\nabla_{X}^{*} F Y-\nabla_{Y}^{*} F X \in \Gamma\left(F\left(D_{2}\right)\right)$,

(ii) $B h(X, F Y)=B h(Y, F X)$,

(iii) $h^{*}(X, F Y)=h^{*}(Y, F X)$,

for any $X, Y \in \Gamma\left(D_{2}\right)$.

Proof. For any $X, Y \in \Gamma(\operatorname{Rad}(T M))$ using (3.3), we have $\tilde{\nabla}_{X} F Y=F \tilde{\nabla}_{X} Y$, applying $F$ to both sides and then using (2.6), (2.13) and (3.1), we get

$$
\nabla_{X} Y+h(X, Y)=F\left(\nabla_{X}^{*} F Y+h^{*}(X, F Y)+h(X, F Y)\right),
$$

for any $X, Y \in \Gamma\left(D_{2}\right)$. Taking the tangential components of above equation both sides, we obtain

$$
\nabla_{X} Y=f \nabla_{X}^{*} F Y+f h^{*}(X, F Y)+B h(X, F Y)
$$

for any $X, Y \in \Gamma\left(D_{2}\right)$. Replacing $X$ by $Y$ and subtracting resulting equation from this equation, we get

$$
\begin{aligned}
{[X, Y]=} & f\left(\nabla_{X}^{* t} F Y-\nabla_{Y}^{* t} F X\right)+f\left(h^{*}(X, F Y)-h^{*}(Y, F X)\right) \\
& -B h(X, F Y)+B h(Y, F X)
\end{aligned}
$$

thus $[X, Y] \in \Gamma\left(D_{2}\right)$ iff $\nabla_{X}^{*} F Y-\nabla_{Y}^{*} F X \in \Gamma\left(F\left(D_{2}\right)\right), B h(X, F Y)=B h(Y, F X)$, $h^{*}(X, F Y)=h^{*}(Y, F X)$, which completes the proof.

From Theorem 4.9 we get the following corollary.

Corollary 4.10. Let $M$ be a GCR-lightlike submanifold of a semi-Riemannian product manifold $(\tilde{M}, \tilde{g}, F)$. Then $D_{2}$ defines a totally geodesic foliation in $M$ iff

(i) $\nabla_{X}^{*} F Y \in \Gamma\left(F\left(D_{2}\right)\right)$,

(ii) $B h(X, F Y)=0$,

(iii) $h^{*}(X, F Y)=0$,

for any $X, Y \in \Gamma\left(D_{2}\right)$. 
Theorem 4.11. Let $M$ be a GCR-lightlike submanifold of a semi-Riemannian product manifold $(\tilde{M}, \tilde{g}, F)$. Then $F\left(D_{2}\right)$ is integrable iff

(i) $g\left(A_{\xi}^{*} F \xi^{\prime}, F Z\right)=g\left(A_{\xi^{\prime}}^{*} F \xi, F Z\right)$,

(ii) $\tilde{g}\left(h^{l}\left(F \xi, \xi^{\prime}\right), \xi^{\prime \prime}\right)=\tilde{g}\left(h^{l}\left(F \xi^{\prime}, \xi\right), \xi^{\prime \prime}\right)$,

(iii) $\tilde{g}\left(h^{s}\left(F \xi, \xi^{\prime}\right), W\right)=\tilde{g}\left(h^{s}\left(F \xi^{\prime}, \xi\right), W\right)$,

(iv) $g\left(A_{N} F \xi, F \xi^{\prime}\right)=g\left(A_{N} F \xi^{\prime}, F \xi\right)$,

for any $Z \in \Gamma\left(D_{0}\right), \xi, \xi^{\prime}, \xi^{\prime \prime} \in \Gamma\left(D_{2}\right), N \in \Gamma(\operatorname{ltr}(T M)), W \in \Gamma\left(L_{2}\right)$.

Proof. Using the definition of GCR-lightlike submanifolds, $F\left(D_{2}\right)$ is integrable iff

$$
\tilde{g}\left(\left[F \xi, F \xi^{\prime}\right], Z\right)=\tilde{g}\left(\left[F \xi, F \xi^{\prime}\right], F \xi^{\prime \prime}\right)=\tilde{g}\left(\left[F \xi, F \xi^{\prime}\right], F W\right)=\tilde{g}\left(\left[F \xi, F \xi^{\prime}\right], N\right)=0,
$$

for any $Z \in \Gamma\left(D_{0}\right), \xi, \xi^{\prime}, \xi^{\prime \prime} \in \Gamma\left(D_{2}\right), N \in \Gamma(\operatorname{ltr}(T M)), W \in \Gamma\left(L_{2}\right)$. Then, from (2.7), (2.8), (2.9) and (2.14) we obtain

$$
\begin{aligned}
& g\left(\left[F \xi, F \xi^{\prime}\right], Z\right)=g\left(\tilde{\nabla}_{F \xi} F \xi^{\prime}-\tilde{\nabla}_{F \xi^{\prime}} F \xi, Z\right)=g\left(\tilde{\nabla}_{F \xi} \xi^{\prime}-\tilde{\nabla}_{F \xi^{\prime}} \xi, F Z\right) \\
&=g\left(A_{\xi}^{*} F \xi^{\prime}-A_{\xi^{\prime}}^{*} F \xi, F Z\right), \\
& g\left(\left[F \xi, F \xi^{\prime}\right], F \xi^{\prime \prime}\right)= g\left(\tilde{\nabla}_{F \xi} F \xi^{\prime}, F \xi^{\prime \prime}\right)-g\left(\tilde{\nabla}_{F \xi^{\prime}} F \xi, F \xi^{\prime \prime}\right) \\
&=g\left(\tilde{\nabla}_{F \xi} \xi^{\prime}, \xi^{\prime \prime}\right)-g\left(\tilde{\nabla}_{F \xi^{\prime}} \xi, \xi^{\prime \prime}\right) \\
&=\tilde{g}\left(h^{l}\left(F \xi, \xi^{\prime}\right), \xi^{\prime \prime}\right)-\tilde{g}\left(h^{l}\left(F \xi^{\prime}, \xi\right), \xi^{\prime \prime}\right), \\
& g\left(\left[F \xi, F \xi^{\prime}\right], F W\right)=g\left(\tilde{\nabla}_{F \xi} F \xi^{\prime}, F W\right)-g\left(\tilde{\nabla}_{F \xi^{\prime}} F \xi, F W\right) \\
&=g\left(\tilde{\nabla}_{F \xi} \xi^{\prime}, W\right)-g\left(\tilde{\nabla}_{F \xi^{\prime}} \xi, W\right) \\
&=\tilde{g}\left(h^{s}\left(F \xi, \xi^{\prime}\right), W\right)-\tilde{g}^{s}\left(h^{s}\left(F \xi^{\prime}, \xi\right), W\right), \\
&=\tilde{g}\left(\tilde{\nabla}_{F \xi} F \xi^{\prime}, N\right)-\tilde{g}\left(\tilde{\nabla}_{F \xi^{\prime}} F \xi, N\right) \\
&=-\tilde{g}\left(F \xi^{\prime}, \tilde{\nabla}_{F \xi} N\right)+\tilde{g}\left(F \xi, \tilde{\nabla}_{F \xi^{\prime}} N\right) \\
&=g\left(A_{N} F \xi, F \xi^{\prime}\right)-g\left(A_{N} F \xi^{\prime}, F \xi\right) .
\end{aligned}
$$

Thus from (4.24)-(4.27), the result follows.

From Theorem 4.11 we obtain the following corollary.

Corollary 4.12. Let $M$ be a GCR-lightlike submanifold of a semi-Riemannian product manifold $(\tilde{M}, \tilde{g}, F)$. The distribution $F\left(D_{2}\right)$ is integrable iff

(i) $\tilde{g}\left(h^{l}\left(F \xi^{\prime}, F Z\right), \xi\right)=\tilde{g}\left(h^{l}(F \xi, F Z), \xi^{\prime}\right)$,

(ii) $g\left(A_{\xi^{\prime \prime}}^{*} \xi^{\prime}, F \xi\right)=g\left(A_{\xi^{\prime \prime}}^{*} \xi, F \xi^{\prime}\right)$,

(iii) $\tilde{g}\left(\xi^{\prime}, D^{l}(F \xi, W)\right)=\tilde{g}\left(\xi, D^{l}\left(F \xi^{\prime}, W\right)\right)$,

(iv) $\tilde{g}\left(h^{*}\left(F \xi, F \xi^{\prime}\right), N\right)=\tilde{g}\left(h^{*}\left(F \xi^{\prime}, F \xi\right), N\right)$,

for any $Z \in \Gamma\left(D_{0}\right), \xi, \xi^{\prime}, \xi^{\prime \prime} \in \Gamma\left(D_{2}\right), N \in \Gamma(\operatorname{ltr}(T M)), W \in \Gamma\left(L_{2}\right)$.

\section{REFERENCES}

[1] Atçeken, M. and Kılıç, E. Semi-invariant Lightlike submanifolds of a semi-Riemannian product manifold, Kodai Math. J., 30(3), 361-378 (2007).

[2] Doğan, B., Şahin, B. and Yaşar, E., Screen generic lightlike submanifolds, Mediterranean Journal of Mathematics, 16(4), 104, 1-21 (2019).

[3] Duggal, K. L. and Bejancu, A., Lightlike submanifolds of semi-Riemannian manifolds and applications, Kluwer Academic Publishers, Dordrecht, (1996).

[4] Duggal, K. L. and Şahin B., Screen Cauchy-Riemann lightlike submanifolds, Acta Math. Hung., 106(1-2), 125-153 (2005). 
[5] Duggal, K. L. and Şahin B., Generalized Cauchy-Riemann lightlike submanifolds of kaehler manifolds, Acta Math. Hung., 112(1-2), 107-130 (2006).

[6] Duggal, K. L. and Şahin, B., Generalized Cauchy-Riemann lightlike submanifolds of indefinite Sasakian manifolds, Acta Math. Hung., 122(1-2), 45-58 (2009).

[7] Duggal, K. L. and Şahin B., Differential geometry of lightlike submanifolds, Birkhäuser, Basel, (2010)

[8] Gupta, R.S. and Sharfuddin, A., Generalised Cauchy-Riemann lightlike submanifolds of indefinite kenmotsu manifolds, Note di Matematica, 30(2), 49-60 (2011).

[9] Gupta, R.S., Upadhyay, A. and Sharfuddin, A., Generalised Cauchy-Riemann lightlike submanifolds of indefinite cosymplectic manifolds, An. Ştiinţ. Univ. "Al. I. Cuza" Iaşi. Mat. (N.S.), 58(2), 381-394 (2012).

[10] Khursheed Haider S. M., Thakur, M. and Advin, Screen Cauchy-Riemann lightlike submanifolds of a semi-Riemannian product manifold, Int. Electron. J. Geom., 4(2), 141-154 (2011).

[11] Kılıç, E. and Şahin, B., Radical Anti-Invariant Lightlike submanifolds of semi-Riemannian product manifolds, Turkish Journal of Mathematics, 32(4), 429-449 (2008).

[12] Kılıç, E., Şahin, B. and Keleş, S., Screen semi-invariant lightlike submanifolds of a semiRiemannian product manifolds, Int. Electron. J. Geom., 4, 120-135 (2011).

[13] Kumar, R., Jain, V. and Nagaich, R. K., GCR-lightlike product of indefinite sasakian manifolds, Advances in Mathematical Physics, Article ID 983069, 1-13 (2011).

[14] Kumar, R., Kumar, S. and Nagaich, R. K., Integrability of distributions in GCR-lightlike submanifolds of indefinite Kaehler manifolds, Communications of the Korean Mathematical Society, 27(3), 591-602 (2012).

[15] Kumar, S., Kumar, R. and Nagaich, R. K., GCR-lightlike submanifolds of a semi-Riemannian product manifold, Bull. Korean Math. Soc., 51(3), 883-899 (2014).

[16] Kupeli, D. N., Singular Semi-Riemannian Geometry, Kluwer, Dordrecht, 366 (1996).

[17] (Önen) Poyraz, N., Golden GCR-Lightlike submanifolds of golden semi-Riemannian manifolds, Mediterranean Journal of Mathematics, 17(5), 1-16 (2020).

[18] Perktaş, S. Y., Kılıç, E. and Keleş, S., Warped product submanifolds of Lorentzian paracosymplectic manifolds, Arabian Journal of Mathematics, 1(3), 377-393 (2012).

[19] Senlin, X. and Yilong, N., Submanifolds of product Riemannian manifold, Acta Mathematica Scientia, 20(2), 213-218 (2000).

[20] Şahin, B., Lightlike submanifolds of indefinite quaternion Kaehler manifolds, Demonstratio Mathematica, 40(3), 701-720 (2007).

Çukurova University, Department of Mathematics, 01330, Adana, Turkey

Email address: nonen@cu.edu.tr 